NASA Technical Memorandum 107002

AIAA-95-2998

\title{
Effects of Initial Conditions on a Single Jet in Crossflow
}

D.S. Liscinsky and B. True

United Technologies Research Center

East Hartford, Connecticut

J.D. Holdeman

Lewis Research Center

Cleveland, Ohio

Prepared for the

31st Joint Propulsion Conference and Exhibit cosponsored by AIAA, ASME, SAE, and ASEE San Diego, California, July 10-12, 1995

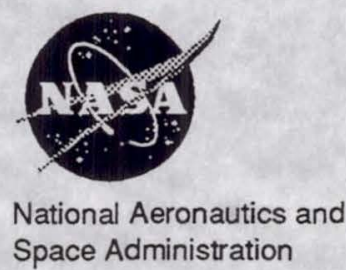




\title{
Effects of Initial Conditions on a Single Jet in Crossflow
}

\author{
D.S. Liscinsky* and B. True* \\ United Technologies Research Center \\ East Hartford, CT 06108 \\ J.D. Holdeman ${ }^{\dagger}$ \\ NASA Lewis Research Center \\ Cleveland, $\mathrm{OH} 44135$
}

\begin{abstract}
An experimental investigation of the effects of jet inlet flow conditions has been conducted for the isothermal mixing of a single jet injected into a crossflow. Jet penetration and mixing was studied using planar Mie scattering to measure time-averaged jet mixture fraction distributions. The effects of 'passive' control methods such as jet 'tabs' and jet swirl are reported. Mixing effectiveness, determined using a spatial unmixedness parameter based on the variance of the mean jet concentration distributions, was compared to a baseline case of a round jet injected into a uniform crossflow. All results are compared at a jet-to-mainstream momentum-flux ratio of 8.5. In the near-field, the mixing rates are similar to, or less than, the baseline configuration using this measure of mixedness. None of the tested configurations appear to significantly augment mixing within a downstream distance of 3 diameters of an equivalent-area round orifice.
\end{abstract}

\section{Nomenclature}

$\alpha \quad$ angle between longest dimension of orifice and axial direction

$A_{j} \quad$ orifice area

$A_{\mathrm{m}}$ cross-sectional area of mainstream duct at injection location

$\mathrm{AR}$ orifice aspect ratio $=\mathrm{L} / \mathrm{W}$

d orifice diameter

$C_{\text {avz }} \quad\left(m j / m_{m}\right) /\left(1+m_{j} / m_{m}\right)$

$C_{d}$ orifice discharge coefficient

J jet-to-mainstream momentum-flux ratio $=\left(\rho_{j} V_{j}^{2}\right) /\left(\rho_{m} U_{\text {main }}^{2}\right)$

$L \quad$ long dimension of orifice

$\mathbf{m}_{\mathbf{j}} \quad$ mass flow of the jet

$m_{m} \quad$ mass flow of the mainstream

$p_{j} \quad$ density of the jet

$\rho_{\mathrm{m}}$ density of the mainstream

$\mathrm{U}_{\mathrm{g}}$ spatial unmixedness parameter (see Eq. 1)

* Research Scientist, Member AIAA

** Senior Laboratory Technician

$\dagger$ Senior Research Engineer, Associate Fellow AIAA

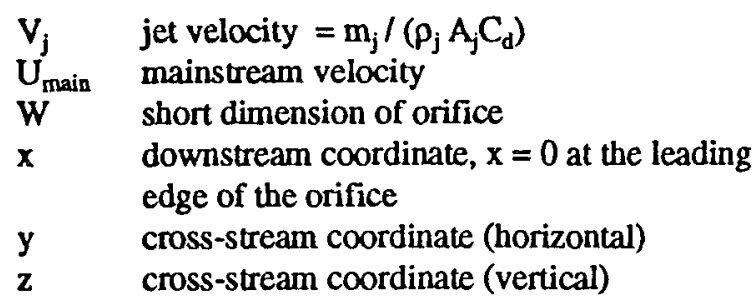

Introduction

Crossflow mixing is used in many applications where the objective is to rapidly obtain a homogeneous mixture of the injectant and mainstream. The mixing process is affected by a number of parameters and optimization of the process in a confined duct has been the topic of several recent investigations ${ }^{1-23}$. The challenge to further increase mixing rates without a corresponding increase in pressure drop is being met by efforts to exploit 'passive' techniques to enhance mixing.

The use of 'passive' methods to control mixing has been widely studied in axisymmetric free jets ${ }^{2427}$. Orifice shape has been demonstrated to augment mass entrainment rates in axisymmetric systems exhausting into quiescient surroundings. In those studies non-circular, low aspect ratio orifices were found to increase mass entrainment rates by factors of 2 over circular jets. In addition to the use of orifice shape alone, the use of a 'delta tab' (a triangular protrusion into the flow at the exit of the jet) has also been found to augment mass entrainment in axisymmetric free jets. In both of these 'passive' configurations the increased entrainment is accompanied by a phenomena called axis switching in which the jet contracts in the direction of the major axis and expands along the minor axis so that after some downstream distance the two axes have interchanged. The interchange is not due to rotation and results in a significant increase in mass entrainment rate.

The increased mixing rates of these techniques can be attributed to generation of vorticity. In the case of a noncircular orifice, the nonuniform boundary layer around the 
circumference of the orifice generates primarily azimuthal vorticity. With the proper placement of tabs streamwise vorticity can also be introduced. These two different phenomena are referred to as $\omega_{\theta}$-induced dynamics (azimuthal) and $\omega_{x}$-induced dynamics (streamwise) ${ }^{24}$. The azimuthal and streamwise vortical structures alone, and in concert, have been shown to promote mixing and spreading in axisymmetric configurations.

Besides vorticity generation, the use of swirl is another 'passive' method to affect mixing. Many experimental studies have shown the dramatic effects of swirl on aflowfield, in particular the ability to change entrainment rate ${ }^{28}$. In general the effects of swirl are increasingly dramatic as the degree of swirl increases, however pressure drop also increases. Swirling flows are characterized by the ratio of radial to axial pressure gradients which can range from streamlines which merely rotate to streamlines which rotate and recirculate. Therefore entrainment can be easily varied by the degree of swirl, particularly in an axisymmetric configuration.

In this investigation the jet inlet conditions were changed by adding 'tabs' and swirl to the jet The jet was then injected into a crossflow and the mixing rate measured. In a crossflow configuration the shear layer between the jet and crossflow generates significant vorticity at the point of injection. Whether the 'passive' techniques which were so success- ful in axisymmetric free jets could add significant vorticity in a crossflow configuration was the subject of the study. For simplification only a single jet has been used. The effects on jet penetration and mixing are now discussed.

\section{Experimental}

The mixing experiments were performed in a $127 \mathrm{~mm}$ $\mathrm{x} 127 \mathrm{~mm}$ horizontal windtunnel with provision for jet injection through one wall as shown in fig. 1. The air for the crossflow was supplied by a blower attached to the tunnel inlet with an $203 \mathrm{~mm}$ diameter flexible duct. The inlet/settling section was $432 \mathrm{~mm} \times 432 \mathrm{~mm}$ and contained a dense "furnace" filter to distribute the flow, followed by a honeycomb and a pair of wire-mesh $50 \%$ open screens for flow conditioning. The $432 \mathrm{~mm} \times 432 \mathrm{~mm}$ cross section then contracted on all four sides by a $3^{\text {rd }}$ order polynomial to the $127 \mathrm{~mm} \times 127 \mathrm{~mm}$ test section. The crossflow/mainstream velocity variation across the test section was less than $5 \%$. Turbulence intensity was $1 \%$.

The jet enters the tunnel through a $3.2 \mathrm{~mm}$ thick bottom wall of the test section. The other three walls of the test section are $3.2 \mathrm{~mm}$ thick plate glass. The jet flow originates in a $102 \mathrm{~mm} \times 102 \mathrm{~mm} \times 127 \mathrm{~mm}$ plenum attached to the bottom wall. The bottom wall contains a removeable plate in which openings are machined to serve as the orifice itself, or to flushmount inserts, such as swirlers.

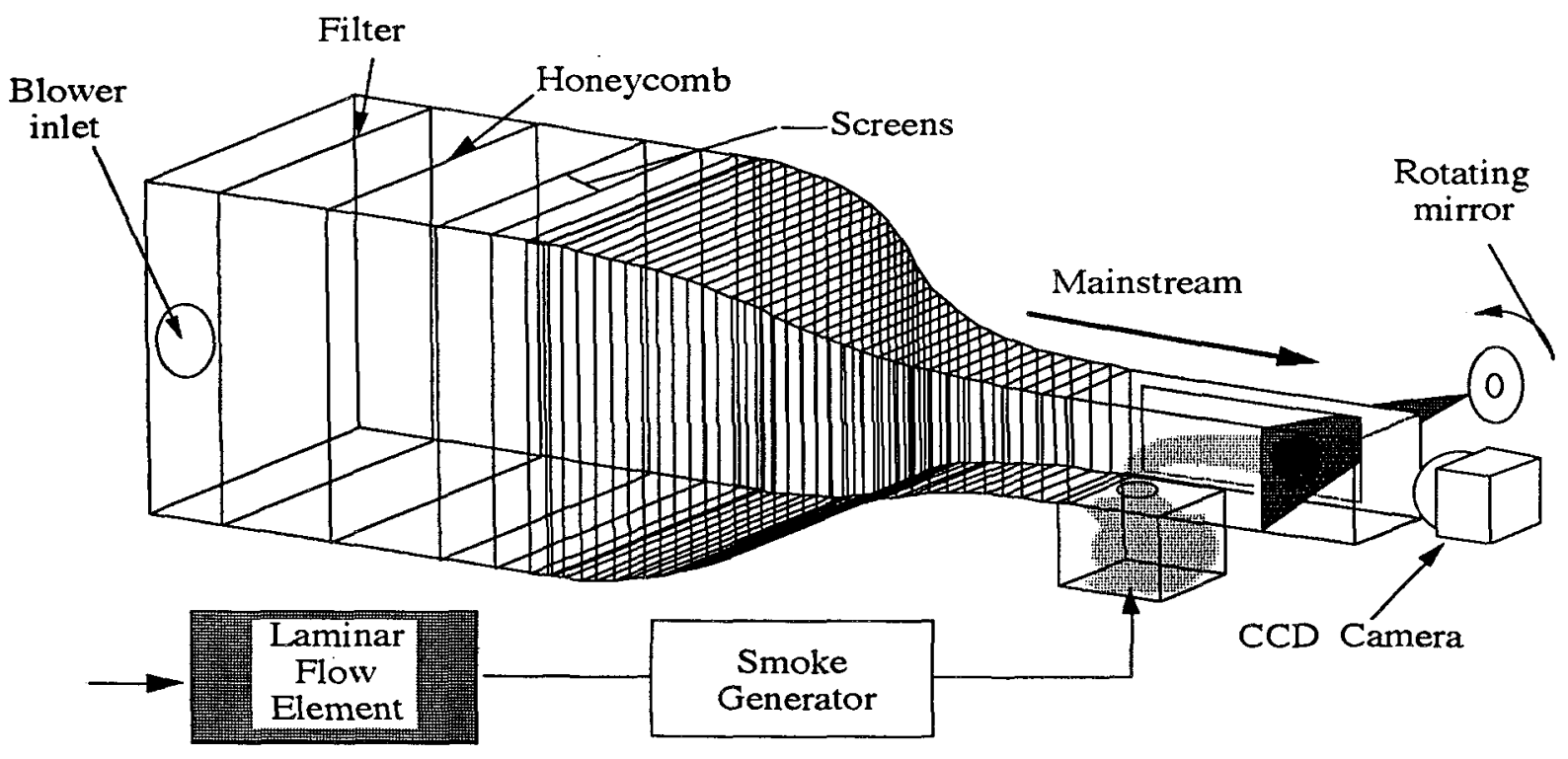

Figure 1: Experimental Configuration used to Measure Planar Concentration Distributions 
Jet-to-mainstream momentum-flux ratio $(J)$ was set at $8.5 \pm 0.2$ for each of the configurations using an $A C_{d}$ that was determined experimentally. This $J$ was chosen so that the round jets would have a trajectory that roughly followed the midpoint of the tunnel and avoided wall contact. Jet mass flow was held constant and the tunnel flow was adjusted to fix $J$, therefore the jet-to-mainstream mass-flow ratio was slightly different for some configurations. Typical jet Reynolds number was 24000 .

' Tab ' Configurations:

The 'tab' was a triangular protrusion into the jet flow at the inlet plane parallel to the wall. The apex angle of the tab was $90 \mathrm{deg}$ with each adjacent side equal to $4.76 \mathrm{~mm}$. Tabs were placed on a round orifice with a diameter of $19.0 \mathrm{~mm}$ and the circular ends of a slot which had an aspect ratio of 2:1. The physical area of the orifices without tabs was 284 sq. mm. Addition of the tabs reduced the physical area by about $4 \%$. The tested configurations are shown in Table 1.

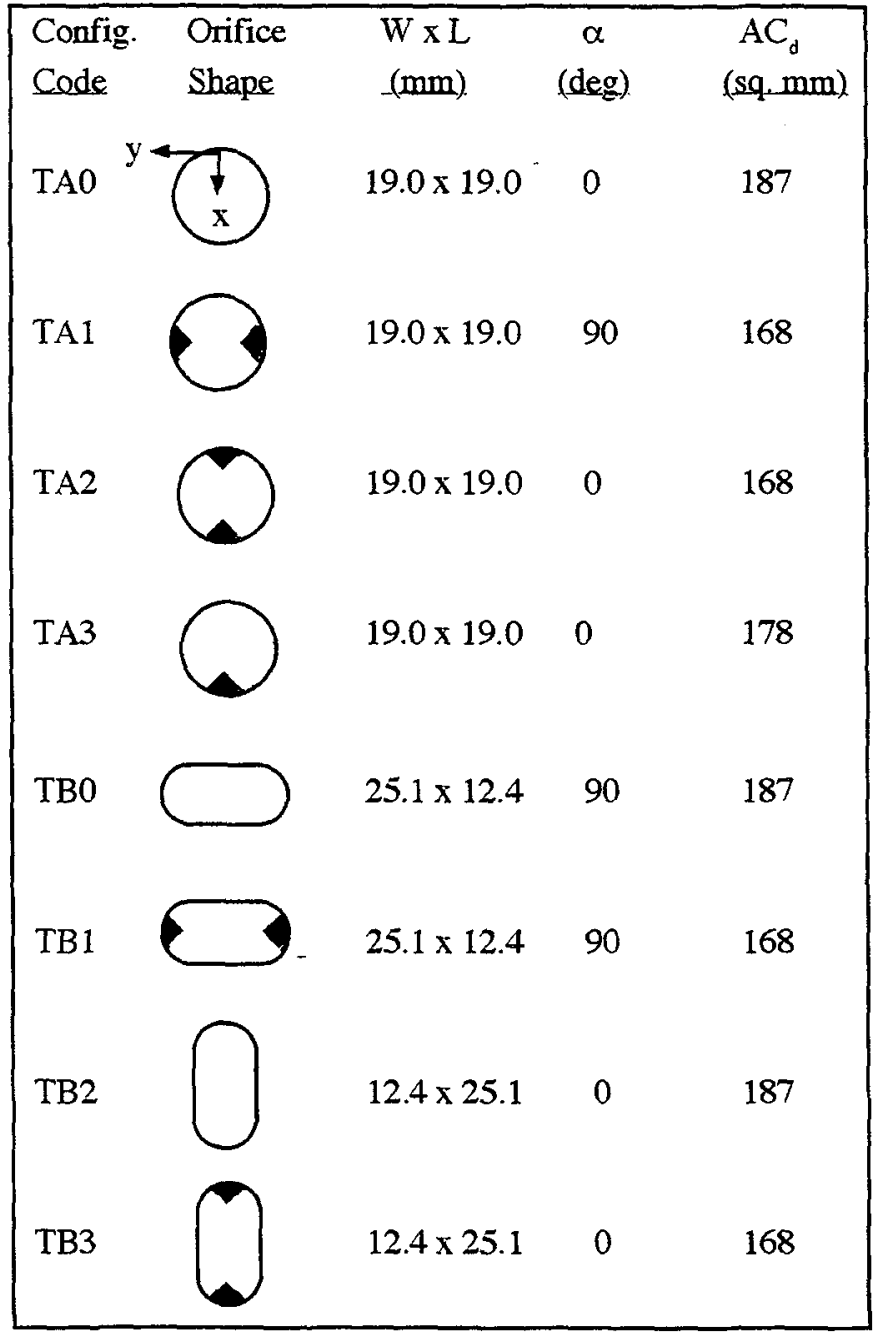

Table 1: ' Tab ' Configurations
Swirler Configurations:

The effect of swirl was studied using stereolithography to fabricate the two swirler configurations shown in figs. 2a and $2 \mathrm{~b}$. Each configuration had 12 flat vanes which were subsequently angled to provide 6 different swirl numbers. The actual swirl number was not measured, but is estimated from the vane angle and tabulated in Table 2 . In the first swirler series (A) $19.0 \mathrm{~mm}$ vanes were connected between a $25.4 \mathrm{~mm}$ OD and a $6.4 \mathrm{~mm}$ center hub, shown with a vane angle of 0 deg in fig. $2 \mathrm{a}$. In the second swirler series (B) $8.9 \mathrm{~mm}$ vanes surrounded a $12.7 \mathrm{~mm}$ diameter center jet, shown with a vane angle of $0 \mathrm{deg}$ in fig. $2 \mathrm{~b}$. Vane angle was varied as shownin Table 2 along with the experimentally measured discharge coefficients.

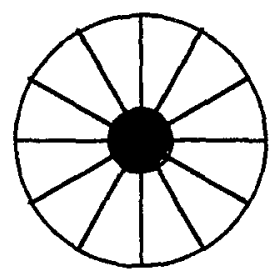

Figure 2a: Swirler A (center hub)

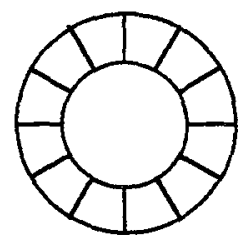

Figure 2b: Swirler $B$ (center jet)

\begin{tabular}{|c|c|c|c|}
\hline $\begin{array}{l}\text { Config. } \\
\text { Code }\end{array}$ & $\begin{array}{c}\text { Swirl } \\
\text { Number }\end{array}$ & $\begin{array}{l}\text { Vane } \\
\text { Angle }\end{array}$ & $\begin{array}{l}\mathrm{AC}_{d} \\
\text { (sq.mm) }\end{array}$ \\
\hline SAO & 0 & 0 & 290 \\
\hline SAl & 0.05 & 5 & 290 \\
\hline $\mathrm{SA} 2$ & 0.1 & 10 & 290 \\
\hline $\mathrm{SA} 3$ & 0.2 & 15 & 290 \\
\hline SA4 & 0.4 & 30 & 271 \\
\hline SA5 & 0.7 & 45 & 187 \\
\hline SB0 & 0 & 0 & 239 \\
\hline SB1 & 0.05 & 5 & 239 \\
\hline SB2 & 0.1 & 10 & 239 \\
\hline SB3 & 0.2 & 15 & 239 \\
\hline SB4 & 0.4 & 30 & 239 \\
\hline SB5 & 0.7 & 45 & 213 \\
\hline
\end{tabular}

Table 2: Swirler Configurations

Mie scattering was the primary diagnostic used to optically measure jet mixture fraction distributions in planes parallel and perpendicular to the duct axis. The planar digital imaging technique (see Ref. 14) is applied by marking the jet flow with an oil aerosol ( $\mu \mathrm{m}$ sized particles). A light sheet 
( $0.4 \mathrm{~mm}$ thick) is created using a $2 \mathrm{~W}$ argon-ion laser and a rotating mirror. The flow field was illuminated to acquire planes of the end-on view, by passing the light sheet througb planes perpendicular to the injection wall ( $y-z$ plane) at various axial locations. A thermo-electrically cooled CCD camera fitted with standard $35 \mathrm{~mm}$ lenses was used to record the scattered light intensity. For the side views the camera was focussed through the side window on the illuminated plane. For the end-on view the camera was located inside the duct $450 \mathrm{~mm}$ downstream of the orifice midpoint. The camera was programmed to make exposures coincident with the sweep of the beam through the flow field. The mean concentration distributions were acquired over 15 seconds and represent the time-average intensity of about 2000 instantaneous distributions which were then digitized in a $380 \times 380$ pixel format (pixel size $=0.3 \mathrm{~mm} \times 0.3 \mathrm{~mm} \times 0.4 \mathrm{~mm}$ ) and sent to a computer for storage. The scattered light intensity is proportional to the number of particles in the measurement volume. If only one of two streams is marked (in this study the jet fluid), the light intensity of the undiluted marked fluid represents mole fraction unity.

\section{Results and Discussion}

Mean concentration distributions for round orifice configuration with and without tabs are shown in Fig. 3. The distributions are end-on views showing $y-z$ planes at 5 downstream locations. A 32-level color scale is used to represent contours of jet mixture fraction from 0 to 1 , where pure mainstream fluid colored red $=0$, and pure jet fluid colored dark purple $=1$. In each plot the lowest contour representing jet mixture fraction $0-0.031$ has been colored white instead of red for better illustration. The box surrounding the distribution at each station represents a $76.2 \mathrm{~mm} \times 76.2 \mathrm{~mm}$ area which starts at the bottom wall of the duct and is centered on the midpoint of the orifice. Note that the concentration distribution was obtained over the entire duct but is not shown. A plan view of the orifice configuration is shown in the upper left corner of the first location for each data set. The mainstream flow direction is from top to bottom for the orifice schematic. In all cases the jet-to-mainstream momentum-flux ratio is 8.5 .

The top row of distributions represents the baseline case of a round orifice without tabs. Development of a pair of counterrotating vortices characteristic of a jet in crossflow is indicated as the jet enters the mainstream and is carried downstream. Jet penteration increases and the plume spreads with increasing downstream distance. The mixing field that results from tabs placed perpendicular to the mainstream flow direction is shown in the second row of distributions. The effect of the tabs is to slightly increase jet penetration and decrease spreading. Decay of the maximum concentration appears to be very similar to the baseline case. Rotation of the configuration so that the tabs are aligned with the mainstream flow is shown in the third row. The effect of the aligned tabs is to decrease jet penetration and increase spreading. Again decay of the maximum concentration appears to be very similar to the baseline configuration at $x / d \geq 2$. In the fourth row the upstream tab has been removed from the aligned tab configuration. Each downstream distributions is very similar to the baseline case indicating that the upstream tab in configuration TA2 is primarily responsible for the decrease in penetration and increase in spreading observed in the distributions for TA2.

In a two-stream mixing problem the fully mixed concentration is defined by the jet-to-mainstream mass-flow ratio. A measure of the mixing rate can be obtained by comparing the jet mixture fraction distribution at any downstream plane to the fully mixed value. In order to quantify mixing rates and compare the results of other studies the authors ${ }^{13}$ developed a measure of unmixedness based on the variance of the concentration distribution, defined as spatial unmixedness:

$$
\mathrm{U}_{\mathrm{s}}=\frac{\mathrm{c}_{\mathrm{var}}}{\mathrm{c}_{\mathrm{avg}}\left(1-\mathrm{c}_{\mathrm{avg}}\right)}
$$

where,

$$
\begin{aligned}
c_{\mathrm{var}} & =\frac{1}{\mathrm{~m}} \sum_{\mathrm{i}=1}^{\mathrm{m}}\left(\overline{\mathrm{c}}_{\mathrm{i}}-\mathrm{c}_{\mathrm{avg}}\right)^{2} \\
& =\text { spatial concentration variance } \\
\overline{c_{\mathrm{i}}} & =\text { time-average concentration at a pixel } \\
\mathrm{c}_{\mathrm{avg}} & =\text { fully mixed concentration }
\end{aligned}
$$

$\mathrm{U}_{\mathrm{s}}=0$ corresponds to a perfectly mixed system, and $\mathrm{U}_{\mathrm{s}}=1$ a perfectly segregated system. The denominator is the maximum concentration fluctuation that can occur at the specified fully mixed concentration. Normalizing by this factor allows $U_{S}$ values to be compared regardless of the jet to mainstream mass-flow ratio of the system. Therefore, this parameter allows comparison of the relative mixing effectiveness of each configuration reported herein and comparison to other configurations with different mass flow ratios.

Spatial unmixedness is plotted in fig. 4 as a function of downstream position for the round 'tab' configurations shown previously in fig. 3 . The unmixedness curve is started at the trailing edge of the orifice $(x / d=1.0)$ to eliminate any error in computing the fully mixed concentration before all of the jet fluid is injected. The data from 10 data planes is plotted including those shown in fig. 4. The curves indicate mixing rates which become almost asymptotic after an initially rapid decay, however the difference between the rates is insignifi- 


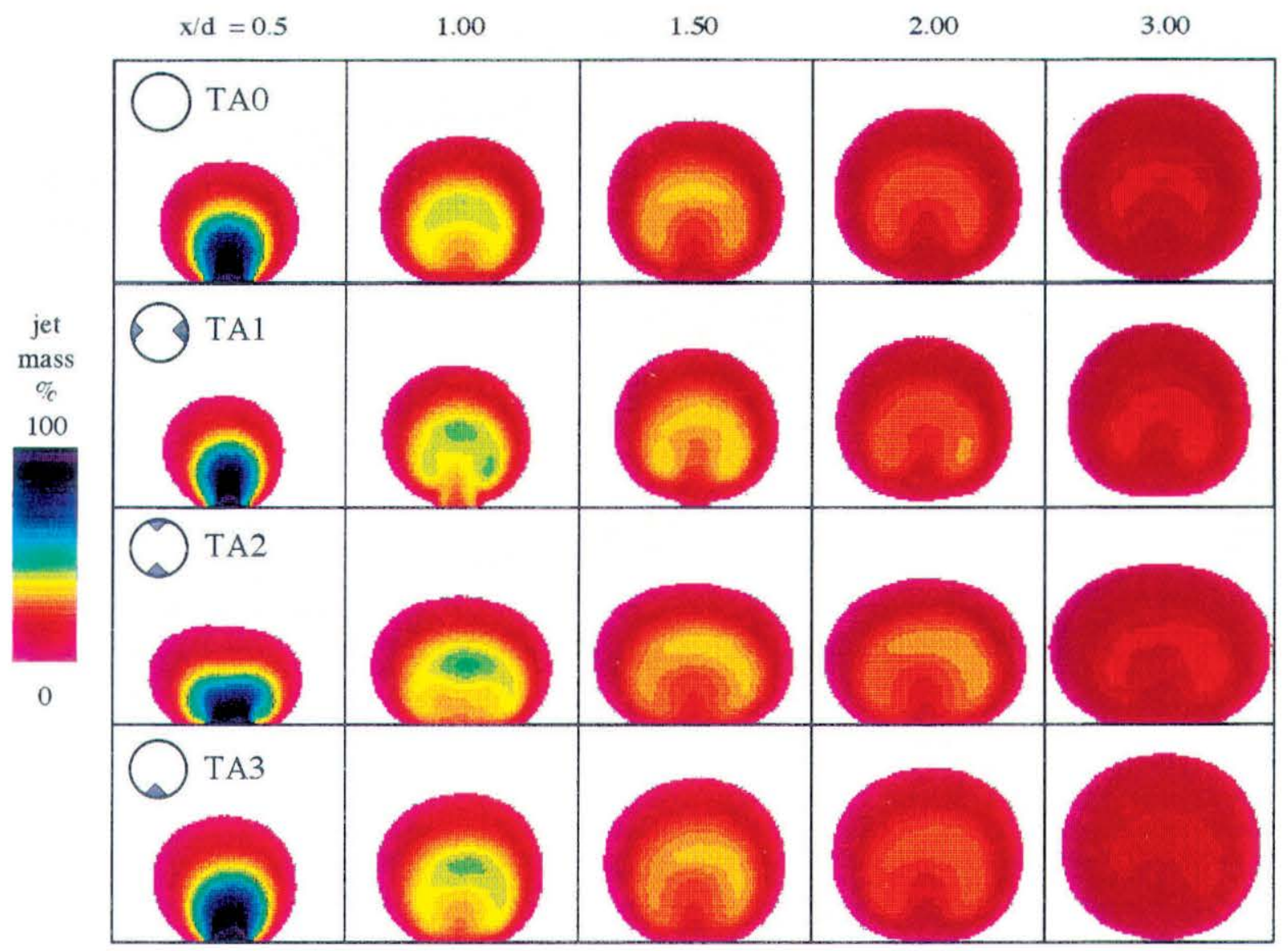

Figure 3: Concentration Distributions of the Round Orifice ' Tab ' Configurations shown in Table 1

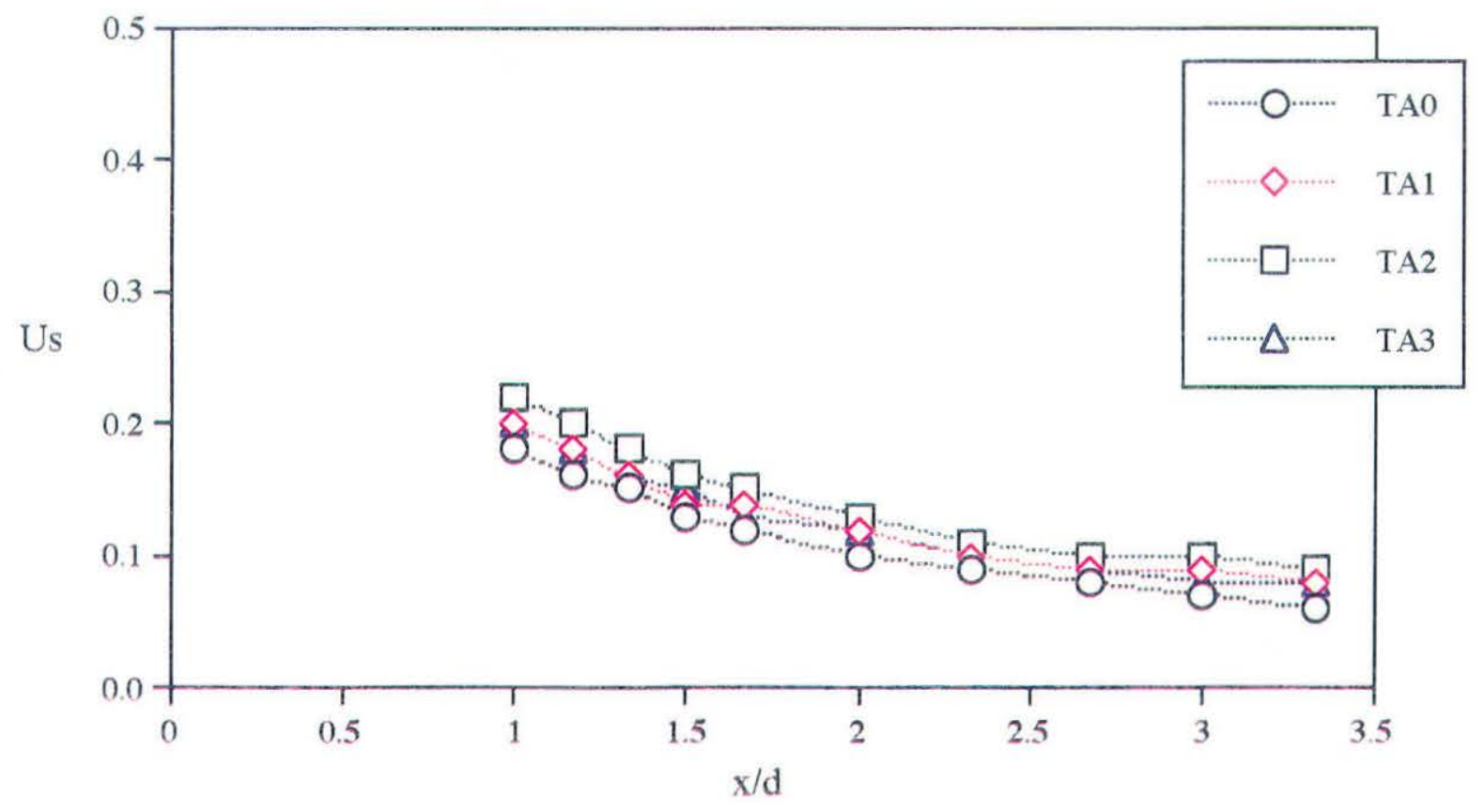

Figure 4: Mixing Rate of the Round Orifice ' Tab ' Configurations shown in Table 1 

cant. The initial rapid mixing rate is attributed to the development of the pair of counterrotating vortices caused shear between the jet and mainstream which leads to rapid local entrainment. However once the jet trajectory bends to be parallel to the mainstream the process slows and the fully mixed condition is slowly approached. The ability of the 'tabs' to augment or sustain the mixing rate is not observed.

In fig. 5 the distributions of the $2: 1$ slots with and without tabs are shown as a function of non-dimensionalized downstream position where " $\mathrm{d}$ " is $19.0 \mathrm{~mm}$ (the diameter of the equivalent area round orifice, configuration TA0). The distribution resulting from the slot oriented transverse to the mainstream, shown in the first row, appears very similar to the round orifice (TA0) shown previously in fig. 3. Penetration and mixing are qualitatively similar although the strength of the vortex pair appears weaker than the round baseline configuration. With the addition of tabs to both ends of the slot jet penetration is decreased and the vortex pair appears weaker. An increase in spreading occurs but the maximum concentration of jet fluid at $x / d=3$ is higher in comparison to the slot without tabs. In the third row the slot is aligned with the mainstream. Penetration is increased compared to transverse slot but mixing appears similar after a slow start due to the extra length of this alignment. The addition of tabs to this configuration is shown in the last row. The tabs decrease penetration but increase spreading. Compared to the baseline, the mixing at $x / d=3$ appears less complete for both of the slot configurations with tabs.

The unmixedness plots for the 4 slot configurations of fig. 5 are shown in fig. 6 . The curves are in agreement with the qualitative assessment of the distributions afforded by fig. 5 . The addition of tabs clearly does not augment the mixing rate in the case of the 2:1 slot. In addition the negative effect of the tab is more significant in the case of the $2: 1$ slot compared to the addition of the tabs to a round orifice.

Concentration distributions of the swirler configurations for the swirlers with a central hub (series A) are shown in fig. 7. Four of the six vane angles that were tested are shown as a function of non-dimensionalized downstream position using a value of $19.0 \mathrm{~mm}$ for $d$ (the diameter of the round orifice, configuration TA0). Although use of this value of $d$ allows this and the subsequent swirler data to be easily compared to the previously presented tab data, note that the diameters of the swirlers are larger than the round orifice (series A diameter is $2.5 .4 \mathrm{~mm}$ and series $B$ is $21.6 \mathrm{~mm}$ ). The first row of distributions is for a swirler with a vane angle of 0 , i.e. no swirl. The jet appears very similar to the baseline round orifice configuration. In the second row the vanes have an angle of $10 \mathrm{deg}$. An asymmetry in the jet appears indicating that a rotation has been imparted to the flow. A vortex pair still develops and penetra- tion does not appear to be drastically changed by the small degree of swirl. Mixing appears similar to the non-swirled case. As the degree of swirl increases in the $30 \mathrm{deg}$ and 45 deg cases, jet penetartion drastically decreases. In addition the swirl appears to prevent the development of the vortex pair. Although lateral spreading increases, entrainment decreases. The lateral asymmetry introduced by the higher degree of swirl appears to cancel the entrainment afforded by the vortex pair in the nonswirled configuration.

In fig. 8 the unmixedness plots for the 6 vane angles are shown as a function of nondimensionalized downstream distance. There is little effect on the mixing rate in the near field for vane angles 0 to 15 , although at the last measurement station the addition of swirl appears to augment the rate. Vane angles above15 deg significantly decrease the mixing rate at $x / d<3$. The rates for the configurations with vane angles less than 15 deg are similar to the baseline round orifice (TA0) at $\mathrm{x} / \mathrm{d}=3$.

The concentration distributions for the swirler configurations that have a center jet (series B) are shown in fig. 9, again plotted as a function of nondimensionalized downstream distance where the value of $d$ is $19.0 \mathrm{~mm}$. The distributions for the $0 \mathrm{deg}$ swirl case are similar to all of the other nonswirled configurations shown previously. As swirl from the $10 \mathrm{deg}$ vanes is added around the center jet as shown in the second row of distributions the symmetry of the jet is preserved and the vortex pair is not weakened as was the case where the entire jet flow is swirled (SA1). As more swirl is added in the 30 deg case the symmetry of the jet is still preserved again unlike the fully swirled case (SA4). Finally with the addition of $45 \mathrm{deg}$ of swirl the jet becomes distorted and development of the vortex pair appears impedded.

Spatial unmixedness of the series B swirlers is shown in fig. 10. The mixing rates are similar except for the $45 \mathrm{deg}$ configuration. The effect of the center jet has been to extend the range of swirl that can be introduced into the jet with a negative effect on mixing performance. Unfortunately the extra swirl does not augment the rate over the nonswirled case. 


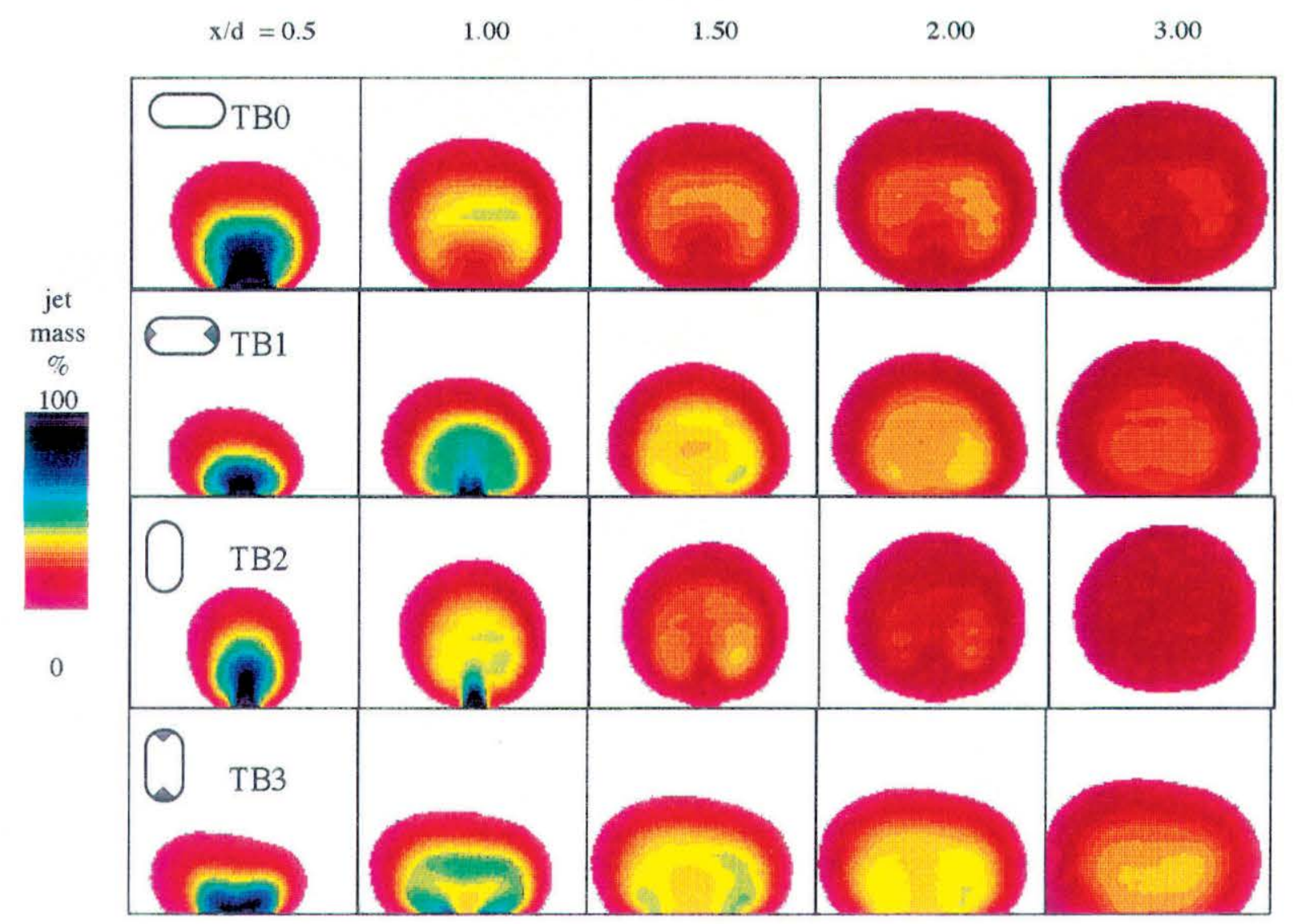

Figure 5: Concentration Distributions of the 2:1 Slot ' $\mathrm{Tab}$ ' Configurations (axial locations are non-dimensionalized by $\mathrm{d}$, the equivalent round orifice diameter)

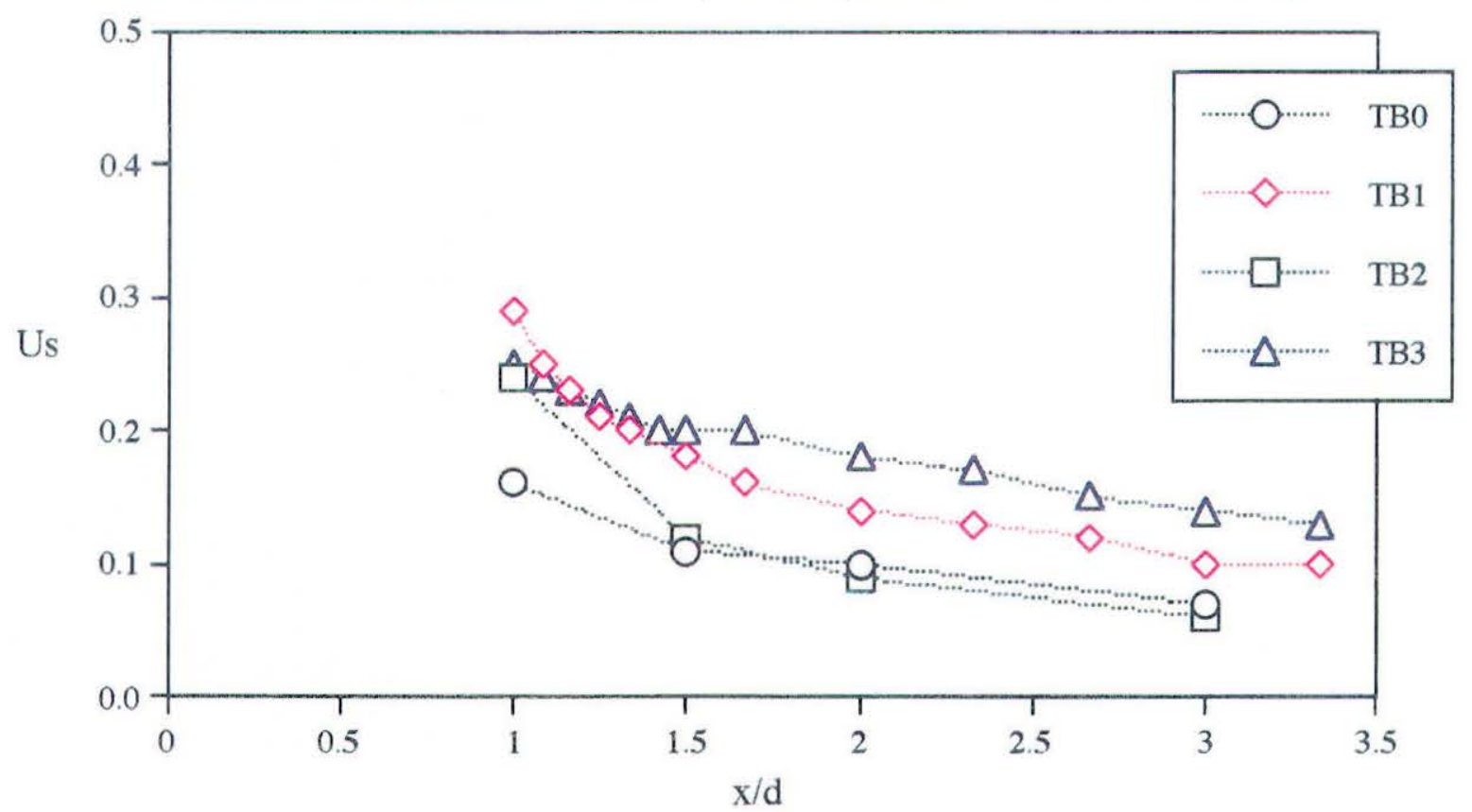

Figure 6: Mixing Rates of the 2:1 Slot ' $T a b$ ' Configurations

(axial locations are non-dimensionalized by $\mathrm{d}$, the equivalent round orifice diameter) 
. 


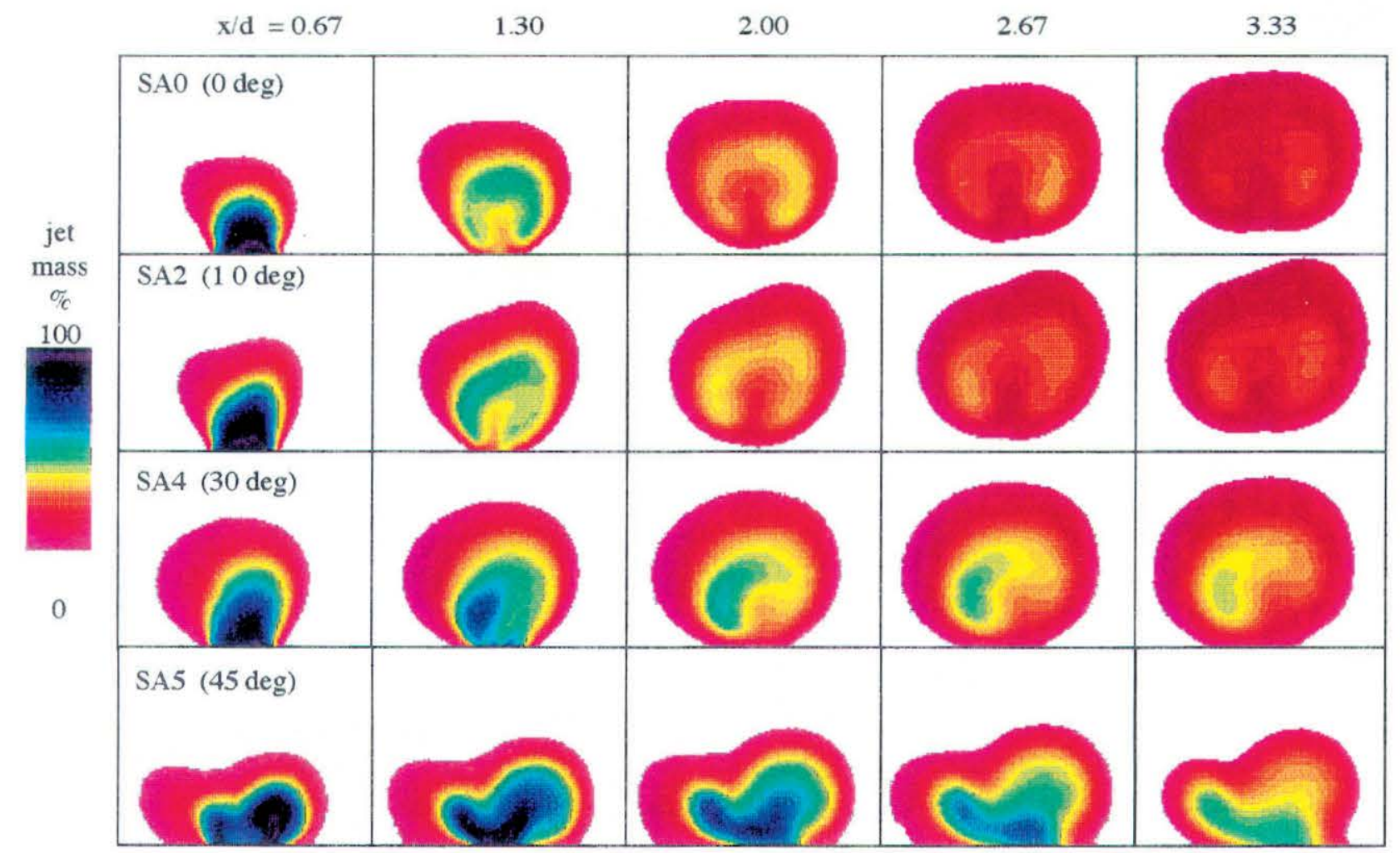

Figure 7: Concentration Distributions of Swirler A Configurations (axial locations are non-dimensionalized by d, the equivalent round orifice diameter)

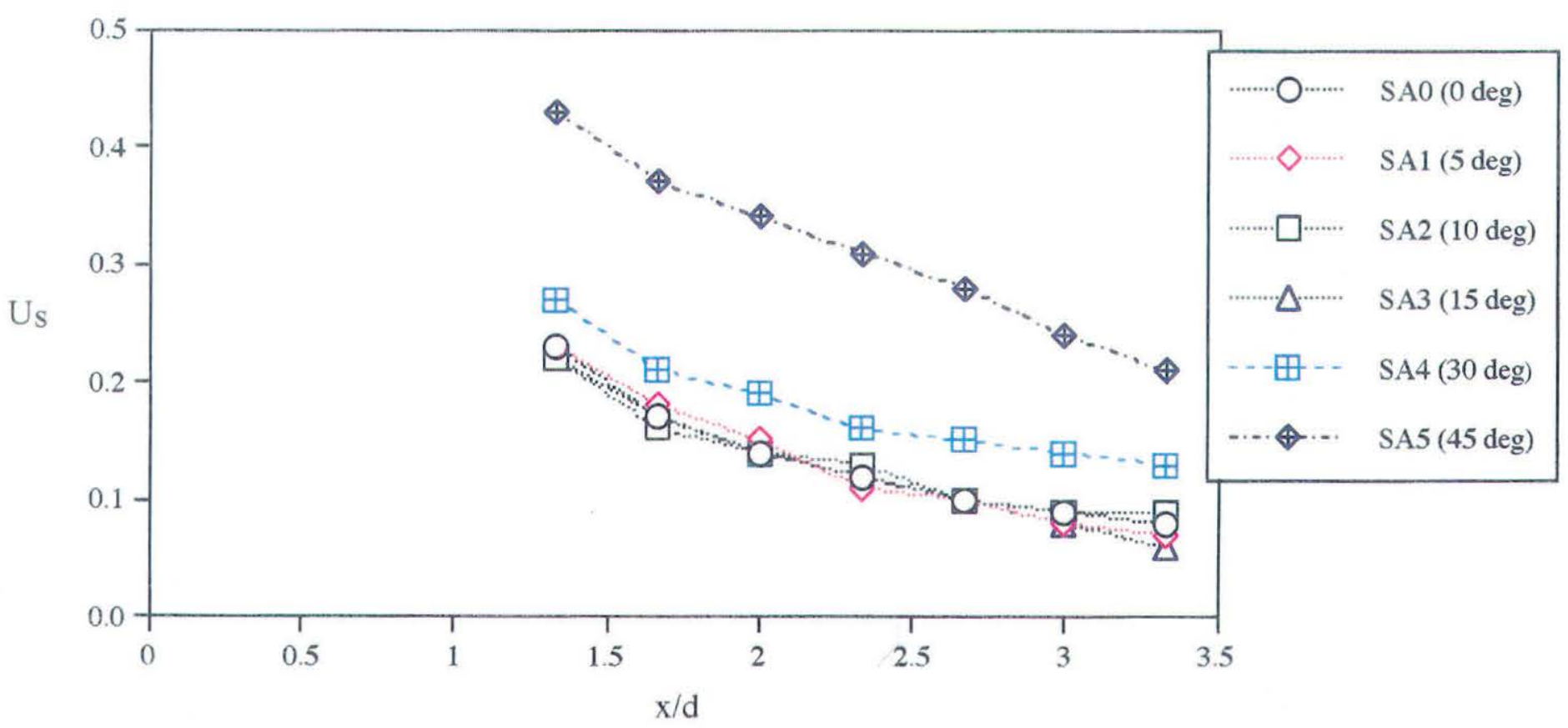

Figure 8: Spatial Unmixedness of Swirler A Configurations (axial locations are non-dimensionalized bv $\mathrm{d}$. the eauivalent round orifice diameter) 


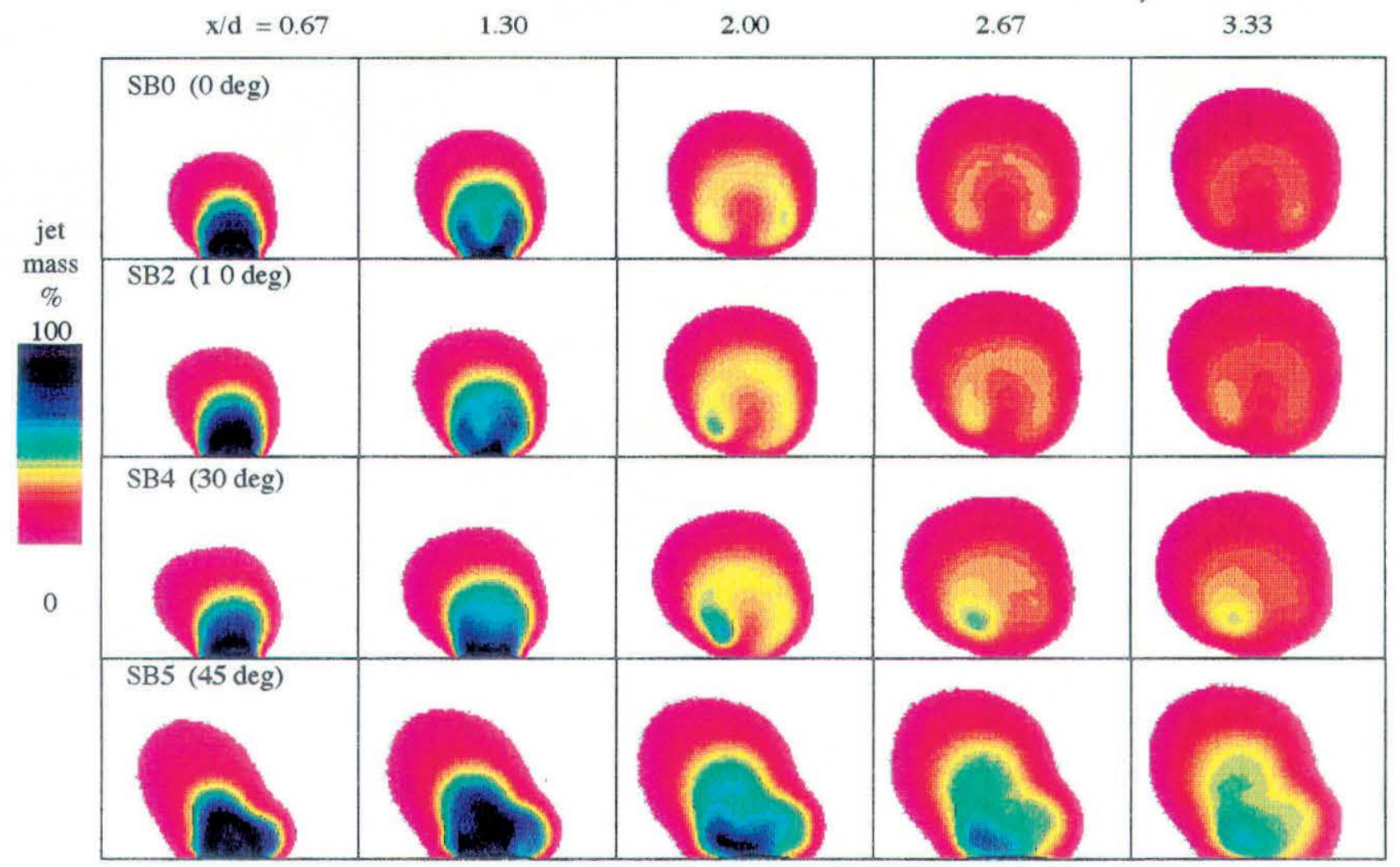

Figure 9: Concentration Distributions of Swirler B Configurations

(axial locations are non-dimensionalized by $\mathrm{d}$, the equivalent round orifice diameter)

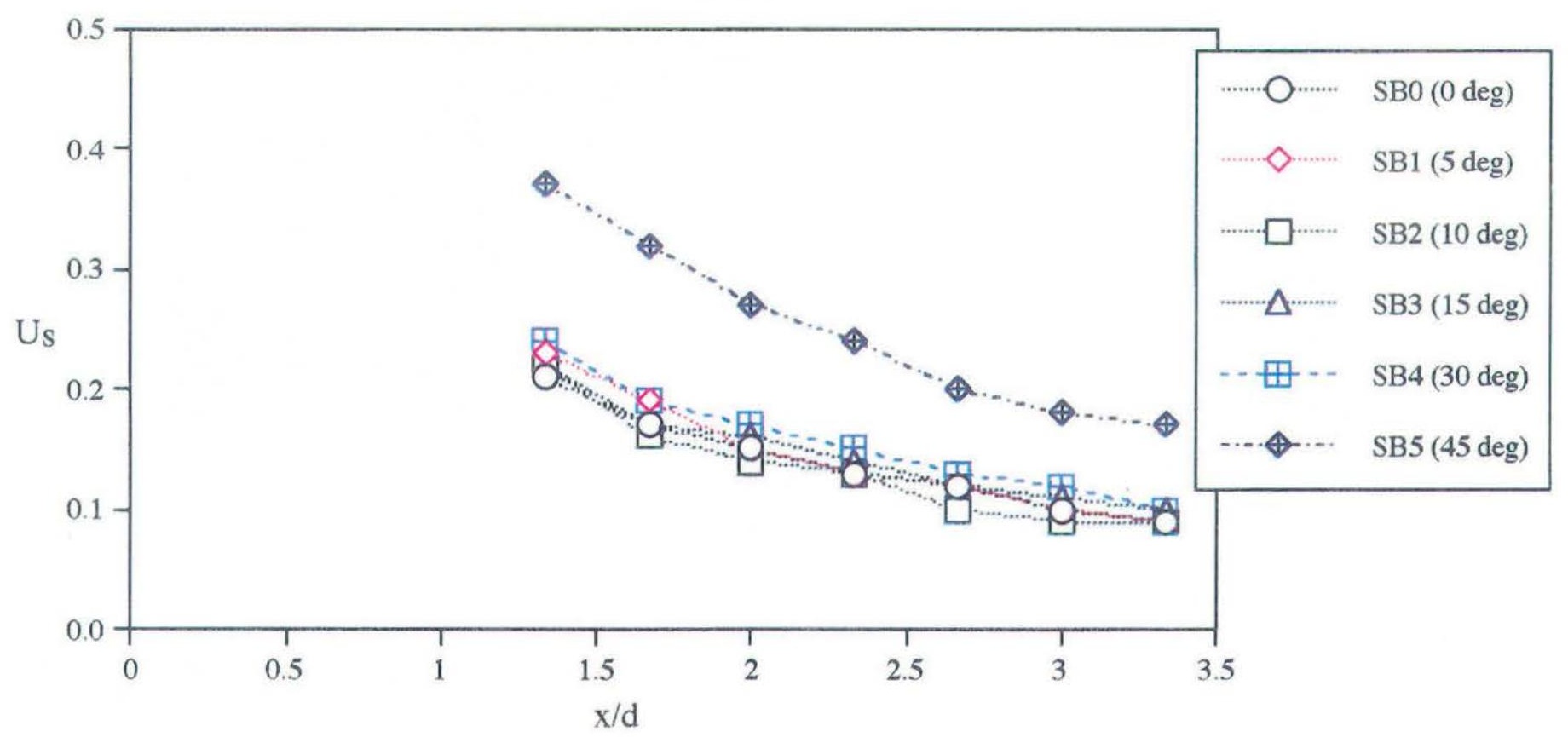

Figure 10: Spatial Unmixedness of Swirler B Configurations (axial locations are non-dimensionalized by $\mathrm{d}$, the equivalent round orifice diameter) 


\section{Conclusions}

Two 'passive' control methods that significantly improve mass entrainment rates in axisymmetric free jet configurations have been experimentally investigated in a jet-in-crossflow configuration. None of the tested configurations significantly augmented mixing performance within a downstream distance of 3 orifice diameters compared to the baseline configuration of a round orifice. However the following effects were observed:

For the 'tab' configurations -

- Orientation of a 'tab' relative to the crossflow streamlines can modify jet penetration

- The effect of a 'tab' on slot configurations is more significant than on round configurations

- The ' tab' configurations did not generate significant vorticity compared to that generated by the crossflow

For the swirler configurations -

- Jet penetration decreases with increasing swirl number

- Moderate swirl number does not augment mixing performance

- Addition of a non-swirled center jet increases jet penetration relative to a fully swirled configuration, but jet penetration is not increased relative to the round baseline configuration

\section{References}

1. Bain, D.B., Smith, C.E., and Holdeman, J.D., "CFD Assessment of Orifice Aspect Ratio and Mass Flow Ratio on Jet Mixing in Rectangular Ducts," AIAA 94-0218, (also NASA TM 106434), Jan. 1994.

2. Bain, D.B., Smith, C.E., and Holdeman, J.D., "CFD Mixing Analysis of Axially Opposed Rows of Jets Injected into a Confined Crossflow," accepted for publication in Journal of Propulsion and Power, (see also AIAA 93-2044, and NASA TM 106179, June 1993).

3. Bain, D.B., Smith, C.E., and Holdeman, J.D., "CFD Mixing Analysis of Jets Injected from Straight and Slanted Slots into Confined Crossflow in Rectangular Ducts," AIAA 92-3087, (also NASA TM 105699), July 1992.
4. Doerr, Th., Blomeyer, M., and Hennecke, D.K., "Optimization of Multiple Jets Mixing with a Confined Crossflow," ASME Paper 95-GT-313, International Gas Turbine and Aeroengine Congress \& Exposition, Houston, June 1995.

5. Doerr, Th. and Hennecke, D.K., "The Mixing Process in the Quenching Zone of the Rich-Lean Combustion Concept," AGARD-PEP 81st Symposium of Fuels and Combustion Technology for Advanced Aircraft Engines, 1993.

6. Hatch, M.S., Sowa, W.A., Samuelsen, G.S., and Holdeman, J.D., "Jet Mixing Into a Heated Cross Flow in a Cylindrical Duct: Influence of Geometry and Flow Variations," Journal of Propulsion and Power, 11 (3), pp. 393402, May/June 1995 (see also AIAA 92-0773 and NASA TM 105390).

7. Hatch, M.S., Sowa, W.A., Samuelsen, G.S., and Holdeman, J.D., "Influence of Geometry and Flow Variations on NOFormation in the Quick Mixer of a Staged Combustor," accepted for publication in Journal of Engineering for Gas Turbines and Power, (also NASA TM 105639, July 1992).

8. Holdeman, J.D., "Mixing of Multiple Jets with a Confined Subsonic Crossflow," Prog. Energy Combust. Sci., vol. 19, pp. 31-70, 1993 (see also AIAA 91-2458 and NASA TM 104412, June 1991).

9. Howe, G.W., Li, Z., Shih, T.I.-P., and Nguyen, H.L., "Simulation of Mixing in the Quick Quench Region of a Rich Burn - Quick Quench Mix- Lean Bum Combustor," AIAA-91-0410, 1991.

10. Kroll, J.T., Sowa, W.A., Samuelsen, G.S., and Holdeman, J.D., "Optimization of Circular Orifice Jets Mixing into a Heated Crossflow in a Cylindrical Duct," AIAA 93-0249, (also NASA TM 105984), Jan. 1993.

11. Liscinsky, D.S., True, B., and Holdeman, J.D., "Crossflow Mixing of Noncircular Jets," accepted for publication in Journal of Propulsion and Power, (see also AIAA-950732 and NASA TM 106865, Jan. 1995).

12. Liscinsky, D.S., True, B., and Holdeman, J.D., "Mixing Characteristics of Directly Opposed Rows of Jets Injected Normal to a Crossflow in a Rectangular Duct," AIAA-940217, (also NASA TM 106477), Jan. 1994. 
13. Liscinsky, D.S., True, B., and Holdeman, J.D., "Experimental Investigation of Crossflow Jet Mixing in a RectangularDuct," AIAA 93-2037,(alsoNASA TM 106152), June 1993.

14. Liscinsky, D.S., True, B., Vranos, A., and Holdeman, J.D., "Experimental Study of Cross-Stream Mixing in a Rectangular Duct," AIAA Paper 92-3090, (also NASA TM 106194), July 1992.

15. Oechsle, V.L. and Holdeman, J.D., "Numerical Mixing Calculations of Confined Reacting Jet Flows in a Cylindrical Duct, " AIAA-95-0733, (also NASA TM 106736), Jan. 1995.

16. Oechsle, V.L., Mongia, H.C., and Holdeman, J.D., "Comparison of the Mixing Calculations of the Reacting and Nonreacting Flows in a Cylindrical Duct," AIAA-94 0865, (also NASA TM 106435), Jan. 1994.

17. Oechsle, V L., Mongia, H.C., and Holdeman, J.D., "An Analytical Study of Jet Mixing in a Cylindrical Duct," AIAA 93-2043, (also NASA TM 106181), June 1993.

18. Oechsle, V.L., Mongia, H.C., and Holdeman, J.D., "A Parametric Numerical Study of Mixing in a Cylindrical Duct," AIAA 92-3088, (also NASA TM 105695), July 1992.

19. Smith, C.E., Talpallikar, M.V., and Holdeman, J.D., "A CFD Study of Jet Mixing in Reduced Areas for Lower Combustor Emissions," AIAA Paper 91-2460, (also NASA TM 104411), June 1991.

20. Sowa, W.A., Kroll, J.T., Samuelsen, G.S., and Holdeman, J.D, "Optimization of Orifice Geometry for CrossFlow Mixing in a Cylindrical Duct," AIAA 94-0219, (also NASA TM 106436), Jan. 1994.

21. Talpallikar, M.V., Smith, C.E., Lai, M.C., and Holdeman, J.D., "CFD Analysis of Jet Mixing in Low NOx Flametube Combustors," Journal of Engineering for Gas Turbines and Power, 114, pp. 416, 1992, (also ASME 91-GT-217 and NASA TM 104466, 1991).

22. Vranos, A., Liscinsky, D.S., True, B., and Holdeman, J.D., "Experimental Study of Cross-Stream Mixing in a Cylindrical Duct," AIAA 91-2459, (also NASA TM 105180), June 1991.
23. Zhu, G., and Lai, M.-C., "A Parametric Study of Penetration and Mixing of Radial Jets in Necked-Down Cylindrical Cross-Flow," AIAA-92-3091, July 1992.

24. Ho, C.-M, and Gutmark, E., "Vortex Induction and Mass Entrainment in a Small-Aspect-Ratio Elliptic Jet," Journal of Fluid Mechanics, 179, pp383-405, 1987.

25. Gutmark, E. and Schadow, K.C., "Flow Characteristics of Orifice and Tapered Jets," Physics of Fluids, 30 (11), pp. 3448-3454, November 1987.

26. Quinn, W.R., "On Mixing in an Elliptic Turbulent Free Jet, "Physics of Fluids, A 1(10), pp. 1716-1722, October 1989.

27. Zaman, K.B.M.Q. "Axis Switching and Spreading on an Asymmetric Jet -- Role of Vorticity Dynamics," AIAA 95-0889, Jan. 1995

28. Gupta, A.K., Lilley, D.G., and Syred, N., Swirl Flows, Abacus Press, 1984.

\section{Acknowledgements}

This work was supported by NASA Contract NAS325954, Task Order \#12 and NASA Contract NAS3-27235, Task 1.0.2.6. We would like to thank Dr. William A. Sowa of Pratt \& Whitney for collaboration on the swirler configurations and Lou Ann Ibets of Pratt \& Whitney for final design and fabrication of the swirlers. 


\begin{tabular}{|c|c|c|}
\hline \multicolumn{2}{|c|}{ REPORT DOCUMENTATION PAGE } & $\begin{array}{l}\text { Form Approved } \\
\text { OMB No. 0704-0188 }\end{array}$ \\
\hline \multicolumn{3}{|c|}{$\begin{array}{l}\text { Public reporting burden for this collection of information is estimated to average } 1 \text { hour per response, including the time for reviewing instructions, searching existing data sources, } \\
\text { gathering and maintaining the data needed, and completing and reviewing the collection of information. Send comments regarding this burden estimate or any other aspect of this } \\
\text { collection of information, including suggestions for reducing this burden, to Washington Headquarters Services, Directorate for Information Operations and Reports, } 1215 \text { Jefferson } \\
\text { Davis Highway, Suite 1204, Arlington, VA 22202-4302, and to the Office of Management and Budget, Paperwork Reduction Project (0704-0188), Washington, DC } 20503 \text {. }\end{array}$} \\
\hline 1. AGENCY USE ONLY (Leave blank) & \begin{tabular}{|c|c|} 
2. REPORT DATE & 3. \\
July 1995 &
\end{tabular} & $\begin{array}{l}\text { ID DATES COVERED } \\
\text { echnical Memorandum }\end{array}$ \\
\hline \multicolumn{3}{|c|}{ Effects of Initial Conditions on a Single Jet in Crossflow } \\
\hline $\begin{array}{l}\text { 6. } \text { AUTHOR(S) } \\
\text { D.S. Liscinsky, B. True, and }\end{array}$ & Holdeman & WU-537-02-21-00 \\
\hline $\begin{array}{l}\text { 7. PERFORMING ORGANIZATION NA } \\
\text { National Aeronautics and Sp } \\
\text { Lewis Research Center } \\
\text { Cleveland, Ohio } 44135-31\end{array}$ & $\begin{array}{l}\text { S) AND ADDRESS(ES) } \\
\text { Administration }\end{array}$ & $\begin{array}{l}\text { 8. PERFORMING ORGANIZATION } \\
\text { REPORT NUMBER }\end{array}$ \\
\hline \multicolumn{2}{|c|}{ 9. SPONSORING/MONITORING AGENCY NAME(S) AND ADDRESS(ES) } & $\begin{array}{l}\text { 10. SPONSORING/MONITORING } \\
\text { AGENCY REPORT NUMBER } \\
\text { NASA TM-107002 } \\
\text { AIAA-95-2998 }\end{array}$ \\
\hline
\end{tabular}

\section{SUPPLEMENTARY NOTES}

Prepared for the 31st Joint Propulsion Conference and Exhibit cosponsored by AIAA, ASME, SAE, and ASEE, San Diego, California, July 10-12, 1995. D.S. Liscinsky and B. True, United Technologies Research Center, East Hartford, Connecticut 06108; J.D. Holdeman, NASA Lewis Research Center. Responsible person, J.D. Holdeman, organization code 2650, (216) 433-5846.

12a. DISTRIBUTION/AVAILABILITY STATEMENT

12b. DISTRIBUTION CODE

Unclassified - Unlimited

Subject Category: 07

Available electronically at http://gltrs.grc.nasa.gov/GLTRS

This publication is available from the NASA Center for AeroSpace Information, (301) 621-0390.

13. ABSTRACT (Maximum 200 words)

An experimental investigation of the effects of jet inlet flow conditions has been conducted for the isothermal mixing of a single jet injected into a crossflow. Jet penetration and mixing was studied using planar Mie scattering to measure timeaveraged jet mixture fraction distributions. The effects of 'passive' control methods such as jet 'tabs' and jet swirl are reported. Mixing effectiveness, determined using a spatial unmixedness parameter based on the variance of the mean jet concentration distributions, was compared to a baseline case of a round jet injected into a uniform crossflow. All results are compared at a jet-to-mainstream momentum-flux ratio of 8.5. In the near-field, the mixing rates are similar to, or less than, the baseline configuration using this measure of mixedness. None of the tested configurations appear to significantly augment mixing within a downstream distance of 3 diameters of an equivalent-area round orifice.

14. SUBJECT TERMS

Dilution; Jet mixing flow; Gas turbine; Emissions; Combustion chamber

\begin{tabular}{|c|c|c|}
\hline $\begin{array}{c}\text { 17. SECURITY CLASSIFICATION } \\
\text { OF REPORT } \\
\text { Unclassified }\end{array}$ & $\begin{array}{c}\text { 18. SECURITY CLASSIFICATION } \\
\text { OF THIS PAGE } \\
\text { Unclassified }\end{array}$ & $\begin{array}{c}\text { 19. SECURITY CLASSIFICATION } \\
\text { OF ABSTRACT } \\
\text { Unclassified }\end{array}$ \\
\hline
\end{tabular}

NSN 7540-01-280-5500

Standard Form 298 (Rev. 2-89) 